\title{
Overview on the Anatomical and Clinical Aspects of Mandibular Prognathism
}

\author{
Ahmed M. S. Hegazy ${ }^{1, ~ *, ~ B a k r ~ A h m e d ~ B a k r ~}{ }^{2}$ \\ ${ }^{1}$ Anatomy Department, Benha Faculty of Medicine, Benha University, Benha City, Egypt \\ ${ }^{2}$ Laser in Dentistry, Dental Consultant, Ministry of Health, Giza City, Egypt \\ Email address: \\ ahmed0562301954@yahoo.com (A. M. S. Hegazy)
}

\section{To cite this article:}

Ahmed M. S. Hegazy, Bakr Ahmed Bakr. Overview on the Anatomical and Clinical Aspects of Mandibular Prognathism. International Journal of Clinical and Developmental Anatomy. Vol. 1, No. 3, 2015, pp. 64-69. doi: 10.11648/j.ijcda.20150103.12

\begin{abstract}
The form and size of the human mandible is subject to considerable variation from the accepted normal. One of the more interesting and rewarding aspects of oral surgery is the operative correction of the Mandibular abnormalities, the category of malformation into which the mandibular deformity falls. In this study we reviewed more than 30 articles to clarify the mandibular prognathism with its effects. Conclusion: Awareness of the normal and abnormal variations of the mandibular anatomy with their causes, deferential diagnosis, prognosis and complications especially mandibular prognathism is very important for the maxillo-facial surgeon to achieve a suitable decision during treatment.
\end{abstract}

Keywords: Prognathism, Mandible, Deformity, Acromegaly, Maxillo-Facial Surgery

\section{Introduction}

The form and size of the human mandible is subject to considerable variation from the accepted normal. One of the more interesting and rewarding aspects of oral surgery is the operative correction of these abnormalities.

It is therefore necessary to devise a classification based upon immediate causes and upon which a rationale of treatment for these cases can be established. A classification upon which both the timing and nature of treatment can be based is as follows:

1. Extreme degrees of normal variation.

- Increased size leading to mandibular prognathism.

- Reduced size leading to mandibular retrognathism.

2. Abnormalities of shape or size occurring as part of a more extensive syndrome.

3. Decrease in size due to localized defect in the mandibular growth center.

- Unilateral.

- Bilateral.

4. Increase in size as a result of over activity of the condylar growth center.

- Unilateral.

- Bilateral.

Bilateral mandibular prognathism often represents the first and most striking physical characteristic of acromegaly; usually, it is also the main reason why patients seek help from orthodontists or maxillo-facial surgeons ${ }^{(1)}$.

\section{Prognathism}

Prognathism is a deformity characterized by an abnormal protrusion of the mandible. The broader part of the lower dental arch lies opposite the narrower portion of the upper arch, causing a malocclusion varying with the degree of the deformity. The protruding chin, with the massive jaw and heavy lips, often results in a very displeasing appearance, which primarily causes the patient to come for surgical aid. It may be due to actual hypertrophy of the bone; errors in the eruption of the teeth; premature extraction of deciduous molars; or to fractures or contracting scars of the face and neck (Figure 1).

Lateral cephalometric radiograph examination (Figure 2) showed massive mandibular prognathism, prominent supraorbital ridges and an enlarged sella turcica. Additionally, magnetic resonance imaging (MRI) scans confirmed the expansively growing tumour mass within and above the sella turcica. Furthermore; the entire calvarian bone was thickened, thus confirming the provisional diagnosis of acromegaly. Endocrinological examination showed increased levels of the insulin-like growth factor-I (IGF-I) $(627.0 \mathrm{ng} / \mathrm{ml}$; norm: 117.0 to $329.2 \mathrm{ng} / \mathrm{ml})$, increased prolactin $(63.69 \mu \mathrm{g} / \mathrm{l}$; norm: 2.10 to $17.7 \mu \mathrm{g} / \mathrm{l})$ and depressed testosterone levels $(0.84 \mu \mathrm{g} / \mathrm{l}$; norm: 2.41 to $8.30 \mu \mathrm{g} / \mathrm{l})$. Ultrasonography showed 
hepatosplenomegaly and an enlarged left kidney. In addition, colonoscopy showed dilatation of the colonic lumen. Ophthalomological screening showed a large bi-temporal visual field defect due to tumor compression of the optic chiasm. The patient underwent transsphenoidal surgery with complete tumor resection $^{(2)}$.

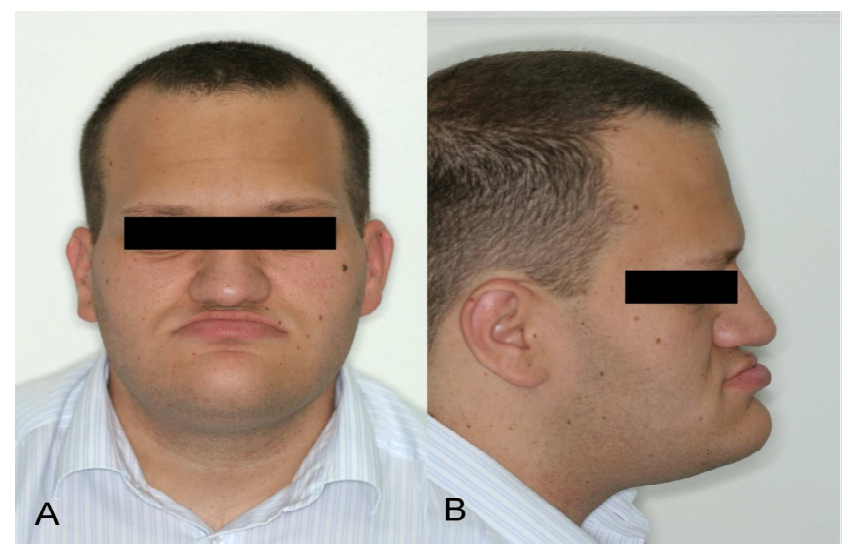

Figure 1. Initial facial photographs (1a: frontal and 1b: lateral view) ${ }^{(37)}$.

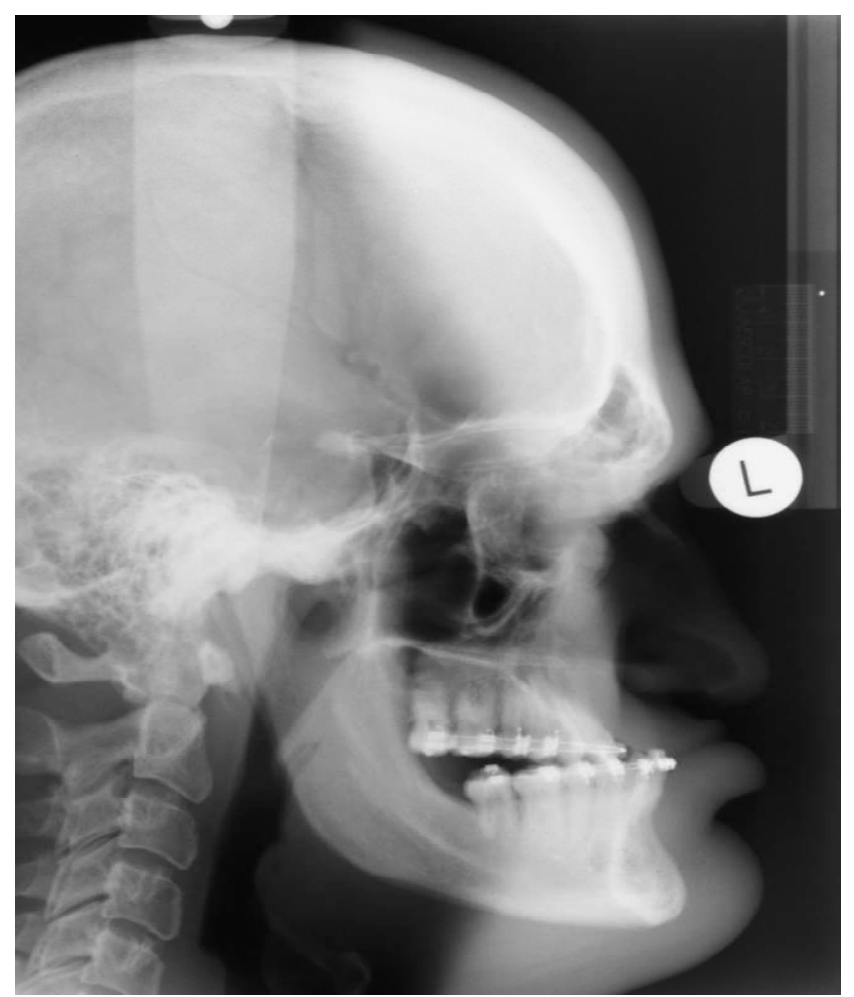

Figure 2. Lateral cephalometric radiograph. ${ }^{(37)}$

Acromegaly and mandibular prognathism were the main reasons for the patient to seek orthodontic treatment. Enlargement of the lips and the tongue, may impair swallowing, chewing and speaking. Frontal bossing, prognathism, macroglossia and an increased size of hands and feet have often been described in the literature as cosmetic changes associated with GH adenomas ${ }^{(3,4,5)}$

Lateral cephalometric radiograph examination showed massive mandibular prognathism, prominent supraorbital ridges and an enlarged sella turcica. Patients with acromegaly usually exhibit enlargement of all parts of the neurocranium and orofacial bones except the maxilla. The mandible usually shows the biggest enlargement, and the ramus is more affected than the body of the mandible ${ }^{(6,7)}$. The enlargement of the sella turcica caused by the tumor expansion of the pituitary gland is a striking manifestation that is detectable on lateral cephalometric radiographs in almost every patient with acromegaly ${ }^{(8)}$.

\subsection{Acromegaly}

Acromegaly (derived from the Greek words "akros", extremities, and "megas", big). This term was proposed by Pierre Marie, a famous French neurologist working in La Salpetrière Hospital, in Paris, who published the first description of the disease and its pathology in 1886. It is used when the disease begins in adulthood ${ }^{(9)}$.

Acromegaly is a rare, insidious, and potentially lifethreatening condition for which there is good, albeit incomplete, treatment that can give the patient additional years of high-quality life ${ }^{(10)}$.

Acromegaly is a rare disease that is responsible for bilateral mandibular prognathism in adults. Excess of growth hormone and local tumor growth of the pituitary gland affect the entire body and increase mortality. In most patients, this disease is diagnosed far too late. Orthodontic and maxillofacial surgeons dealing with orthognatic surgery should be well aware of this disease as patients usually present with them first because of striking mandibular growth. In case of mandibular prognathism in combination with enlargement of lips, nose, tongue, hands and feet followed by visual field defects, sleep apnea, decreased energy and osteoarthritis, every clinician should consider the diagnosis of acromegaly. Although acromegaly is a rare disease, its symptoms are striking and can hardly be misconstrued ${ }^{(11)}$.

Mandibular prognathism normally is the result of an imbalance between the nasomaxillary complex and a prominent mandible. The accompanying dental condition reveals a Class III malocclusion with an anterior cross bite. A compensated inter-dental relationship can be found with labial tipping of the maxillary anterior teeth in conjunction with lingual tipping of the mandibular anterior teeth. However, the vertical dental relationship with either over or open bite conditions varies among patients with mandibular prognathism. Overall, the discrepancies can easily be noticed in the sagittal dental and facial appearance with the prominent vertical feature of greater lower anterior facial height $^{(12)}$.

Researchers have identified a gene on the $\mathrm{X}$ chromosome, GPR101, which was overexpressed 1000-fold more than normal in a genetic study of 43 patients affected by sporadic or inherited gigantism that manifested during childhood or adolescence. This duplication was not evident in patients who began abnormal growth at age 9 or 10, but only in those who started to grow excessively before the age of 3. In a separate analysis of 248 patients with sporadic acromegaly, a mutation in the GPR101 gene was found in about $4 \%$ of cases. Nainggolan L. Gene Discovery in Giants 
Could Shed Light on Human Growth ${ }^{(13)}$.

Acromegaly is an acquired disorder related to excessive production of growth hormone $(\mathrm{GH})$ and characterized by progressive somatic disfigurement (mainly involving the face and extremities) and systemic manifestations. The prevalence is estimated at 1:140,000-250,000. It is most often diagnosed in middle-aged adults (average age 40 years, men and women equally affected). Due to insidious onset and slow progression, acromegaly is often diagnosed four to more than ten years after its onset. The main clinical features are broadened extremities (hands and feet), widened thickened and stubby fingers, and thickened soft tissue. The facial aspect is characteristic and includes a widened and thickened nose, prominent cheekbones, forehead bulges, thick lips and marked facial lines. The forehead and overlying skin is thickened, sometimes leading to frontal bossing. There is a tendency towards mandibular overgrowth with prognathism, maxillary widening, tooth separation and jaw malocclusion. The disease also has rheumatologic, cardiovascular, respiratory and metabolic consequences which determine its prognosis. In the majority of cases, acromegaly is related to a pituitary adenoma, either purely $\mathrm{GH}$-secreting $(60 \%)$ or mixed. In very rare cases, acromegaly is due to ectopic secretion of growth-hormone-releasing hormone (GHRH) responsible for pituitary hyperplasia. The clinical diagnosis is confirmed biochemically by an increased serum $\mathrm{GH}$ concentration following an oral glucose tolerance test (OGTT) and by detection of increased levels of insulin-like growth factor-I (IGF-I).

Assessment of tumor volume and extension is based on imaging studies. Echocardiography and sleep apnea testing are used to determine the clinical impact of acromegaly ${ }^{(11)}$.

Diagnosis of acromegaly as one of the responsible causes of bilateral mandibular prognathism in adults Laboratory studies used in the diagnosis of growth hormone (GH)/IGF-I excess include the following:

Oral glucose: To determine the extent to which the patient can suppress GH concentration after the consumption of oral glucose.

GH: Clearly elevated GH levels $(>10 \mathrm{ng} / \mathrm{mL})$ after oral glucose, combined with the clinical picture, secure the diagnosis of acromegaly.

IGF-I: Elevated IGF-I values in a patient whose symptoms prompt appropriate clinical suspicion almost always indicate GH excess.

Imaging studies include the following:

Magnetic resonance imaging (MRI): To image pituitary adenomas.

Computed tomography (CT) scanning: To evaluate the patient for pancreatic, adrenal, and ovarian tumors secreting GH/GHRH; use chest CT scans to evaluate for bronchogenic carcinoma secreting GH/GHRH.

Radiography: To demonstrate skeletal manifestations of GH/IGF-I excess ${ }^{(14)}$.

\subsubsection{Prognosis}

Because of the small number of people with gigantism, mortality and morbidity rates for this disease during childhood are unknown.

In acromegaly, a severe disease that is often diagnosed late, morbidity and mortality rates are high, particularly as a result of associated cardiovascular, cerebrovascular, and respiratory disorders and malignancies. ${ }^{[1]}$

Because IGF-I is a general growth factor, somatic hypertrophy in acromegaly occurs across all organ systems. Associated complications include the following: $:^{(15)}$

- Acromegalic heart

- Increased muscle and soft tissue mass

- Increased kidney size

- Articular overgrowth of synovial tissue and hypertrophic arthropathy

- Joint symptoms, back pain, and kyphosis: Common presenting features

- Thick skin

- Hyperhidrosis (often malodorous)

- Carpal tunnel syndrome and other entrapment syndromes

- Macroglossia: May result in sleep apnea

Cerebral aneurysm and increased risk of cerebrovascular accident: Less common. ${ }^{(16)}$

\subsection{Effect of Mandibular Prognathism on Masticatory Muscles Volume}

Several investigators have reported significant associations between masticatory muscle size and skeletal craniofacial form $(17,18,19,20)$ while others have found very few correlations between the size of jaw-closing muscles and craniofacial morphology. ${ }^{(21)}$

Strictly speaking, even when the differences in measuring methods are taken into account, there has been no agreement on the association between craniofacial form and the size of related muscular structures ${ }^{(22)}$

To understand biomechanical relationships between hardand soft tissue structures, we believe that it is indispensable to examine the structural association between muscles and the adjacent local skeletal sites on which muscle forces are exerted, rather than examining correlations between the overall craniofacial skeletal structure and the size of masticatory muscles within a traditional cephalometric paradigm. Mandibular prognathism, a gross skeletal deformity of the craniofacial area often requires orthognathic surgery. Because the surgical approach necessitates invasion into the mandibular ramus and gonial region, an understanding of the association between the morphology of the skeletal and muscular components of craniomandibular structures is indispensable in achieving successful treatment and post-treatment stability. In addition, such an understanding will provide meaningful insight into the principles that account for relationships between form and function of the craniomandibular apparatus ${ }^{(23)}$

To study relationships between form and function of the craniomandibular apparatus we use:

1) Examine computerized Tomography (CT) images of the craniofacial structure in adult patients with mandibular prognathism; 
2) Based on CT's three-dimensionality, investigate whether the temporal and masseter muscle volumes correlate with transverse head dimensions;

3) Investigate whether the masseter muscle volume correlates with the size of the zygomatico-mandibular skeletal sites, the form of the antegonial region, and orientation of the masseter muscle;

4) Investigate whether the masseter muscle orientation has influence on the inclination of the zygomaticomandibular skeletal sites ${ }^{(24)}$.

Kitai et al ${ }^{(24)}$ found in their study significant positive correlations were found among temporal muscle crosssectional area, masseter muscle cross-sectional area, temporal muscle volume, and masseter muscle volume. Only the volume data on the temporal and masseter muscle were used for subsequent analyses, because the volume data correlated highly with the cross-sectional area data.

The temporal and masseter muscle volume showed significant positive correlation with the widths of the bizygomatic arch and the temporal fossa but not with cranium width.

There were significant positive correlations among the zygomatic arch cross-sectional area, the mandibular ramus cross-sectional area, and masseter muscle volume.

Masseter muscle volume did not significantly correlate with the gonial angle and the masseter anterior orientation. The masseter zygomatic angle was $95.1+6.2$ degrees, and the masseter antegonial angle was $87.8+8.3$ degrees.

A significant positive correlation was found between the zygomatic arch angle and the antegonial angle.

The temporal and masseter muscle volumes showed significant positive correlation with bizygomatic arch width.

This finding is consistent with the general consensus in previous reports that subjects with strong or thick mandibular elevator muscles have wider transverse head dimensions ${ }^{25.26,}$ $27,28.29,30$ )

The temporal and masseter muscle volumes were significantly correlated with the temporal fossa width but not with the cranium width. These results suggest that the greater bizygomatic arch width for those individuals having large temporal and masseter muscles is not due to the wide cranium but rather to the wide temporal fossa, which is filled primarily with the temporal muscle and partly with the masseter muscle.

Significant positive correlation was found between masseter volume and the cross-sectional areas of the zygomatic arch and the mandibular ramus.

In patients with mandibular prognathism, the bilateral difference in muscle volume would reflect the difference in the spatial anatomy of a skeletal structure and could not predict mandibular skeletal asymmetry ${ }^{(24)}$

\subsection{Effect of Prognathism on the Tongue Volume (Figure 3)}

The morphological relationship between the tongue and maxillo-mandibular structures is an essential element in our understanding of the growth and development of orofacial structures, the etiology of specific types of malocclusions, and occlusal stability after orthodontic or surgical correction of malocclusions. It was hypothesized that the size and form of dental arches were determined by the tongue size $\mathrm{e}^{(31)}$.

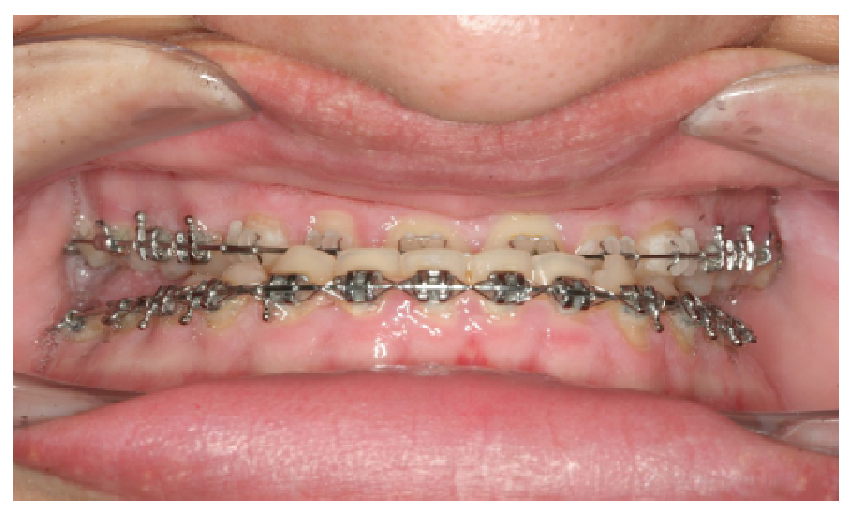

Figure 3. Initial intraoral photograph showing Angle class III malocclusion. (37)

Macroglossia is defined as tongue enlargement due to muscle hypertrophy, tumor or an endocrine disturbance. Pseudo macroglossia, or relative macroglossia, is defined as the tongue being normal in size, but appearing large due to its anatomical reciprocation. The tongue locates forward and is larger than normal in prognathism, and this macroglossia may cause problems such as Class III malocclusion and an open bite ${ }^{(32)}$.

It has also been suggested that an increase in the volume of soft tissues induces the osteogenic reaction at the growth site of the bone. ${ }^{(33)}$

A large tongue has been regarded as a possible cause of mandibular prognathism ${ }^{(34)}$. A relative increase in tongue volume after the mandibular set-back surgery in patients with mandibular prognathism was also suggested as a possible cause of relapse, i.e., decrease in over jet and overbite during the retention period. Although this has formed a basis for the validation of the glossectomy as one of the effective choices for the acquisition of post-treatment occlusal stability the lack of accurate quantitative information on tongue volume has hampered us in giving a definite answer to a simple question of whether mandibular prognathism is accompanied by a large tongue. Indeed, the deciding criteria for glossectomy in adult patients with mandibular prognathism have been based only on subjective visual judgment. ${ }^{(35)}$

Yoo et al. (36) found that the subjects with mandibular prognathism showed tongue volumes similar to those of the control subjects. Hence, it would be logical to assume that a possible increase in relative tongue volume is anticipated in patients with mandibular prognathism who undergo mandibular set-back surgery. The resulting narrowness of the oral cavity may be compensated for by the short-term positional adaptation of the tongue, which was characterized by the simultaneous downward movement of the hyoid bone after mandibular set-back surgery the results obtained in the current study do not support the clinical surmise that patients with mandibular prognathism have larger tongues. Logically, this leads to a possible increase in relative tongue volume in these patients who undergo mandibular set-back surgery. 
Based on the results of the current correlation analysis, it can be speculated that this relative increase in size of the tongue is attuned by a lowering of the mandible to the demands of airway maintenance.

\section{Conclusion}

Awareness of the normal and abnormal variations of the mandibular anatomy with their causes, deferential diagnosis, prognosis and complications especially mandibular prognathism is very important for the maxillo-facial surgeon to achieve a suitable decision during treatment.

\section{References}

[1] Martin Gosau, Corinna Vogel, Antonios Moralis1, Peter Proff, Johannes Kleinheinz and Oliver Driemel; Mandibular prognathism caused by acromegaly - a surgical orthodontic case: Head \& Face Medicine 2009, 5:16.

[2] Martin Gosau, Corinna Vogel, Antonios Moralis1, Peter Proff, Johannes Kleinheinz and Oliver Driemel Head \& Face Medicine 2009, 5:16.

[3] Vitral RW, Tanaka OM, Fraga MR, Rosa EA: Acromegaly in an orthodontic patient. Am J Orthod Dentofacial Orthop 2006, 130:388-90

[4] Jane JR, Laws ER Jr: Surgical management of pituitary adenomas Singapore Med J 2002, 6:318-23.

[5] Chanson P, Salenave S: Acromegaly. Review. Orphanet J Rare Dis 2008, 3:1-17.

[6] Dostalova S, Sonka K, Smahel Z, Weiss V, Marek J: Cephalometric assessment of cranial abnormalities in patients with acromegaly. J Craniomaxillofac Surg 2003, 31(2):80-7.

[7] Chung KC, Buchman SR, Aly HM, Trotman CA: Use of modern craniofacial techniques for comprehensive reconstruction of the acromegalic face. Ann Plast Surg 1996, 36(4):403-8.

[8] Sugata T, Myoken Y, Tanaka S: Acromegaly identified in a patient with a complaint of malocclusion. Oral Surg Oral Med Oral Pathol Oral Radiol Endod 1998, 85(1):44-6.

[9] Daly AF, Rixhon M, Adam C, Dempegioti A, Tichomirowa MA, Beckers A: High prevalence of pituitary adenomas: a cross-sectional study in the province of Liege, Belgium. J Clin Endocrinol Metab 2006, 91:4769-75.

[10] Giustina A, Chanson P, Bronstein MD, Klibanski A, Lamberts $\mathrm{S}$, Casanueva FF, et al. A consensus on criteria for cure of acromegaly. J Clin Endocrinol Metab. Jul 2010;95(7):3141-8.

[11] Philippe Chanson and Sylvie Salenave Orphanet Journal of Rare Diseases 2008, 3:17

[12] Baik HS, Han HK, Kim DJ, Proffit WR. Cephalometric characteristics of Korean Class III surgical patients and their relationship to plans for surgical treatment. Int $\mathrm{J}$ Adult Orthodon Orthognath Surg 2000;15:119-28.

[13] Nainggolan L. Gene Discovery in Giants Could Shed Light on Human Growth Trivellin G, Daly AF, Faucz FR, Yuan B, Rostomyan L, Larco DO, et al. Gigantism and acromegaly due to Xq26 microduplications and GPR101 mutation. N Engl J Med. Dec 18 2014;371(25):2363-74.
[14] Alicia Diaz-Thomas, MD, MPH; Chief Editor: Stephen Kemp, MD, PhD: Gigantism and Acromegaly. medscape jan 6, 2015.

[15] Melmed S, Casanueva FF, Klibanski A, Bronstein MD, Chanson P, Lamberts SW, et al. A consensus on the diagnosis and treatment of acromegaly complications. Pituitary. Sep 2013;16(3):294-302.

[16] Oshino S, Nishino A, Suzuki T, et al. Prevalence of cerebral aneurysm in patients with acromegaly.Pituitary. Jun 2013;16(2):195-201.

[17] Weijs WA, Hillen B (1984). Relationships between masticatory muscle cross-section and skull shape. J Dent Res 63:1154-1157.

[18] Weijs WA, Hillen B (1986). Correlations between the crosssectional area of the jaw muscles and craniofacial size and shape. Am J PhysAnthropol 70:423-431.

[19] Gionhaku N, Lowe AA (1989). Relationship between jaw muscle volume and craniofacial form. J Dent Res 68:805-809.

[20] Benington PC, Gardener JE, Hunt NP (1999). Masseter muscle volume measured using ultrasonography and its relationship with facial morphology. Eur J Orthod 21:659-670.

[21] Hannam AG, Wood WW (1989). Relationships between the size and spatial morphology of human masseter and medial pterygoid muscles, the craniofacial skeleton, and jaw biomechanics. Am JPhys Anthropol 80:429-445.

[22] Shiratsuchi Y, Kouno K, Tashiro H (1991). Evaluation of masticatory function following orthognathic surgical correction of mandibular prognathism. J Craniomaxillofac Surg 19:299-303.

[23] Takada K, Petdachai S, Sakuda M (1993). Changes in dentofacial morphology in skeletal Class III children treated by a modified maxillary protraction headgear and a chin cup: a longitudinal cephalometric appraisal. Eur J Orthod 15:211221.

[24] N. Kitai, Y. Fujii, S. Murakami,S. Furukawa, S. Kreiborg,and K. Takada1 J Dent Res 81(11):752-756, 2002.

[25] Ringqvist M (1973). Isometric bite force and its relation to dimensions of the facial skeleton. Acta Odontol Scand 31:3542 .

[26] Hannam AG, Wood WW (1989). Relationships between the size and spatial morphology of human masseter and medial pterygoid muscles, the craniofacial skeleton, and jaw biomechanics. Am J Phys Anthropol 80:429-445.

[27] Kiliaridis S, Kalebo P (1991). Masseter muscle thickness measured by ultrasonography and its relation to facial morphology. J Dent Res 70:1262-1265.

[28] van Spronsen PH, Weijs WA, Valk J, Prahl Andersen B, van Ginkel FC (1991). Relationships between jaw muscle crosssections and craniofacial morphology in normal adults, studied with magnetic resonance imaging. Eur J Orthod $13: 351-361$

[29] Bakke M, Tuxen A, Vilmann P, Jensen BR, Vilmann A, Toft M (1992). Ultrasound image of human masseter muscle related to bite force, electromyography, facial morphology, and occlusal factors. Scand J Dent Res 100:164-171.

[30] Kiliaridis S (1995). Masticatory muscle influence on craniofacial growth. Acta Odontol Scand 53:196-202. 
[31] Brodie AG (1953). Muscular factors in the diagnosis and treatment of malocclusions. Angle Orthod 23:71-77.

[32] Karamese, M., Akdağ, O., Selimoglu, M.N., Abac1, M., Akatekin, A. and Tosun, Z. Combined Therapy for Mandibular Prognathism: Sagittal Split Osteotomy with Excision of Tongue. Modern Plastic Surgery, 2014, 4, 53-57.

[33] Frankel R, Frankel C (1989). Orofacial orthopedics with the functional regulator. Basel: Karger, pp. 12-18.

[34] Kole H (1965). Results, experience, and problems in the operative treatment of anomalies with reverse overbite (mandibular protrusion). Oral Surg Oral Med Oral Pathol 19:427-450.
[35] (Swanson LT, Murray JE (1969). Partial glossectomy to stabilize occlusion following surgical correction of prognathism. Oral Surg Oral Med Oral Pathol 27:707-715.

[36] E. Yoo, S. Murakamil, K. Takada, H. Fuchihatal, and M. Sakuda. Tongue Volume in Human Female Adults with Mandibular Prognathism. J Dent Res 75(12): 1957-1962, December, 1996.

[37] Martin Gosau,Corinna Vogel, Antonios Moralis, Peter Proff, Johannes Kleinheinz, and Oliver Driemel: Mandibular prognathism caused by acromegaly - a surgical orthodontic case; Head Face Med. 2009; 5: 16.Published online 2009 Aug 6. doi: 10.1186/1746-160X-5-16 PMCID: PMC2741435. 